\title{
La calidad de los procesos de enseñanza de la lectoescritura en Colombia: entre concepciones y prácticas
}

\author{
The quality of the teaching processes of literacy in Colombia: between \\ conceptions and practices
}

\author{
Ferney de Jesús Morales Londoño \\ fercho125mora@hotmail.com \\ Ministerio de Educación de Colombia, Programa Todos Aprender, Colombia
}

\section{RESUMEN}

El propósito de este artículo estuvo orientado a presentar un abordaje teórico conceptual de la calidad en los procesos de enseñanza de la lectoescritura en el nivel de educación básica primaria en Colombia. Se estudió este evento por cuanto, se percibieron grandes dificultades en la lectoescritura de los estudiantes que egresan de la primaria a la secundaria. Para la revisión documental se utilizaron dos niveles, un nivel monográfico dirigido al arqueo de las fuentes para recopilar las ideas de los planteamientos conceptuales de autores como Teberosky y Ferreiro (1982), Lerner (2001), Hernández (2006), entre otros, luego se desarrolló un nivel hermenéutico en el cual se hizo una interpretación de los planteamientos de los autores a partir de la sustentación, vigencia y alcance, de esta manera se plantea la problemática de la lectoescritura en Colombia con relación a los resultados de pruebas saber que se aplican a los estudiantes, el reconocimiento de los lineamientos del MEN para el área de lenguaje y las estrategias como parte de la política de gobierno para el mejoramiento de la calidad de la enseñanza de la lectoescritura en el país. Las reflexiones finales conllevan a resaltar la importancia de construir un marco teórico que explique los factores que inciden en la calidad de la enseñanza de la lectoescritura en el nivel de básica primaria en Colombia.

Palabras clave: Educación; calidad; enseñanza; lectoescritura

\section{ABSTRACT}

The purpose of the following article is to present a conceptual theoretical approach to quality in the processes of teaching literacy at primary school level in Colombia. This event was studied because great difficulties are perceived in the reading and writing of students who graduate from primary to secondary school. The documental review was made in two levels: a monographic one to examine all the documents to know the ideas and the theoretical arguments presented by different authors such as Teberosky and Ferreiro (1988), Lerner (2001), Hernández (2006), among others, and a hermeneuthic level to interpretate the information got from the authors. Initially. The problem of literacy in Colombia is described, having as precedent the results of students in knowledge tests and strategies as part of the policy of the MEN for the improvement of the quality of the teaching of reading and writing in the country. The final reflections are proposed that lead to highlighting the importance of constructing a theoretical framework that explains the factors that affect the quality of literacy teaching at the basic primary level in Colombia.

Key words: Education; quality; teaching; literacy 
INTRODUCCIÓN

La educación es el motor para el desarrollo económico y social de los pueblos. Según Freire (sf cp. Olivera y Orellana, 2005) "la educación es praxis, acción, reflexión del hombre sobre el mundo para transformarlo". Por lo tanto, la educación es el medio que garantiza a las personas ser libres y ejercer con decisión el reconocimiento de otros derechos humanos como el libre desarrollo de la personalidad, la libertad de expresión, la participación democrática y la construcción de ciudadanía.

De esta manera, la educación como motor para la transformación social supera el sistema educativo escolar y se conecta con la realidad social circundante para construir ciudadanía, valoración por la interculturalidad y el respeto por la diversidad. Por tanto, hoy en día hablar de educación de calidad es un tema relevante, por cuanto se hace necesario trabajar en el desarrollo de diferentes políticas educativas, que más allá de garantizar una educación para todos, ayude a garantizar que todos los estudiantes aprendan y que las enseñanzas contribuyan a mejorar su calidad de vida desde sus propios contextos.

Al respecto la UNESCO (2011), expone:

Recibir una educación de calidad a lo largo de toda la vida es un derecho congénito de cada niño, mujer u hombre. Por su parte, la educación, en particular la que se imparte a las niñas y las mujeres, contribuye a la consecución de todos los objetivos del desarrollo (p. 5).

Por ello, garantizar el acceso a una educación de calidad, es una necesidad que apremia a todos los sistemas educativos en el mundo. Todas las personas sin excepción alguna, además, de recibir una educación gratuita, tienen el derecho a recibir una educación de la mejor calidad.

En Colombia hablar de una educación de calidad, es uno de los grandes temas que ocupan la agenda de la política educativa. El propósito ha sumado numerosos esfuerzos en inversión para mejorar la enseñanza en la educación básica y secundaria.

En este sentido, el Ministerio de Educación Nacional (MEN) de Colombia precisa la calidad como "eficacia" y define la educación de calidad como aquella que logra que los estudiantes aprendan lo que deben aprender, es decir, lo que está determinado en los estándares de competencias de las diferentes áreas, y esto debe evidenciarse a través de los resultados de las diferentes pruebas estandarizadas internas y externas que presentan los estudiantes en Colombia.

Así, desde una mirada técnico instrumental, la evaluación de la calidad educativa en Colombia se ve reflejada en los resultados obtenidos por los estudiantes en las pruebas internas SABER y pruebas externas Programme for internacional Student Assessment (PISA). Al respecto, al hacer un análisis de los promedios obtenidos en los últimos 5 años en estas pruebas, se evidencia que, en áreas como matemáticas, lenguaje $\mathrm{y}$ ciencias naturales, se tienen avances significativos en las competencias que evalúan cada una de estas áreas, sin embargo los resultados no son los esperados dentro de las metas de calidad del MEN.

En efecto, con relación a las pruebas SABER, las tablas estadísticas presentadas por el Instituto Colombiano del fomento de la educación superior (ICFES) para el año 2016, indican que el puntaje promedio de las instituciones educativas en los resultados del área de lenguaje está en una escala con media 300 y desviación 80, lo cual representa un bajo desempeño en los tres grados que evalúa la prueba $3^{\circ}, 5^{\circ}$ y $9^{\circ}$. Ilustrando mejor lo 
anterior, las instituciones educativas del país, en una escala de valores de 1,00 - 5,00 (donde 5,00 es el promedio más alto posible), para el grado tercero, el promedio en lenguaje es de 3.13, para quinto grado es de $3.13 \mathrm{y}$ en noveno grado, es de 3.07 .

Respecto a los resultados de las pruebas PISA, de acuerdo al último informe del ICFES 2016, entre 72 países participantes, en el 2015, Colombia ocupó la posición 55 en lectura. Si se comparan los resultados de este año con los del 2006 (donde Colombia realizó su primera participación), se presenta una notable mejora. El área de lectura es donde se observa el mayor progreso, pues en 2015 se obtuvo 40 puntos más en el puntaje promedio.

Desde esta perspectiva, según las pruebas SABER y PISA, es evidente la mejora que Colombia presenta en su calidad educativa, pero el área de lenguaje continúa siendo una de las más problemáticas del sistema educativo colombiano con énfasis en los bajos niveles de competencia en lectura y escritura de los estudiantes.

En consecuencia, el MEN (2013), durante los últimos años ha puesto en práctica diferentes programas y herramientas de calidad para transformar los currículos y promover una mejor enseñanza de las competencias básicas en lenguaje como son la lectura y la escritura. De esta forma, implementa una política de transformación de la calidad educativa mediante el "Programa Todos a Aprender" (PTA), en el cual se establece de forma voluntaria "el Desarrollo Profesional Situado (DPS) a los docentes del nivel educativo primaria de establecimientos oficiales del país que presentan estudiantes con bajos desempeños académicos.

Asimismo, mediante la política educativa del día de la excelencia educativa (Día E), el MEN envía a cada institución educativa una caja de herramientas, que contempla entre muchos otros materiales, los referentes de calidad y los documentos de referencia, a saber: los estándares básicos de competencia, los derechos básicos de aprendizaje (DBA), las matrices de referencia del ICFES, las mallas de aprendizaje y las guías de orientaciones pedagógicas, los cuales tienen como propósito mejorar la calidad de la enseñanza de los docentes en los diferentes ciclos de enseñanza de la educación básica y media.

Desde el programa "Todos a aprender", como parte de los procesos de formación docente, el MEN (2013), realiza un acompañamiento pedagógico a 4700 establecimientos educativos. Este acompañamiento está respaldado por un componente de gestión educativa, comunicación y movilización que involucra toda la comunidad educativa y adicionalmente, consta de un componente pedagógico que provee referentes curriculares, enseñanza, didáctica y materia-les educativos y alternativas de evaluación de aprendizajes en el área de lenguaje y matemáticas.

Además, en este componente de acompañamiento pedagógico se incluyen los procesos de enseñanza en el área de lenguaje, lo cual implica dar prioridad al fortalecimiento de la formación docente en el conocimiento didáctico de contenido para la enseñanza de la lectura y la escritura, la cualificación de las practicas docentes desde el uso de estrategias pedagógicas como el aprendizaje cooperativo de Johnson y Johnson (1999) y la dotación de materiales educativos de alta calidad como apoyo el proceso docente educativo.

A nivel departamental, también las secretarias de educación implementan diferentes estrategias de cualificación docente para el mejoramiento de la enseñanza. En el caso de la secretaria de educación de Antioquia, en los últimos 10 años tiene consolidada una red regional de lenguaje, 
donde se realizan procesos de formación permanente a los maestros en las diferentes subregiones, a través de talleres y seminarios.

Entre los eventos más significativos y reconocidos a nivel departamental está el encuentro con las letras. Allí se profundiza sobre la didáctica en los procesos de la lectura y la escritura, retomando diversas problemáticas que se presentan al momento de su enseñanza, especialmente en los primeros grados de escolaridad.

De acuerdo con varios autores, los principales problemas de lectura y escritura comienzan en los primeros años de escolaridad. Los métodos tradicionales $\mathrm{y}$ mecanicistas comienzan por generar poca motivación en los niños a aprender a leer y a escribir. Por ejemplo un reciente estudio conducido por expertos de la Universidad de la Sabana concluyó que "las dificultades para aprender a leer que experimentan los niños colombianos son consecuencia de métodos erróneos de enseñanza" (Guzmán, 2014).

Guzmán (2014) en su trabajo sobre "Lectura y escritura. Cómo se enseña y se aprende en el aula", expresa que la dificultad de los estudiantes en torno al aprendizaje de la lectoescritura se debe a las formas en como las escuelas y la sociedad entienden el ejercicio de la lectura y la escritura.

Plantea la autora Guzmán, (2014) que:

El aprendizaje de la lectura y la escritura es el más complejo que realiza el ser humano durante toda su vida. Pero los docentes tienen en sus manos la solución para resolver estos conflictos que tanto preocupan a los padres de familia, gracias a una adecuada intervención en el aula de clase (p. 12).

Finalmente, desde el ICFES (2016), se expone que los problemas más relevantes de lectura y escritura se presentan en la educación primaria. Actualmente muchas investigaciones sobre la lectura y la escritura se centran en el fortalecimiento de las competencias lectora y la producción de textos, sin embargo, poco se ha profundizado en el estudio de la calidad de la enseñanza desde la génesis lectura y la escritura, máxime que en los primeros grados de escolaridad $\left(1^{\circ}\right.$ - $3^{\circ}$ ) las dificultades de los niños para aprender a leer y a escribir se reflejan en las altas tasas de repitencia escolar donde se justifica la pérdida del año escolar por la insuficiencia en estos procesos.

\section{MÉTODO}

Este trabajo se orientó a un estudio teórico conceptual sobre la calidad de los procesos de enseñanza de la lectoescritura para lo cual, en el proceso de documentación, se utilizaron los siguientes niveles:

a. Nivel monográfico: el cual se basa fundamentalmente en la descripción temática: recopilación y organización del material existente, sin que el investigador tome posición ni asuma alguna postura en particular. (Hurtado, 2010). De esta manera, la autora antes citada plantea para el desarrollo de este nivel la Técnica de revisión documental Riaceer (Recopilación, identificación de ideas, almacenamiento, categorización, esquema conceptual, enlace y redacción), mediante la cual se revisaron los diferentes aspectos conceptuales planteados por los autores sobre la calidad educativa y la enseñanza de la lectoescritura en Colombia.

b. Nivel herrnenéutico: Este nivel abarca el análisis de la temática donde el investigador crítica y juzga la pertinencia de los planteamientos presentados en el nivel monográfico a partir de su entendímiento, sustentación, alcance. (Hurtado, 2010) 


\section{RESULTADOS Y DISCUSIÓN}

Desde el punto de vista de autores como Bernal y otros (2015), la "calidad" se concibe como un concepto propio del contexto que lo requiera, y del momento en que éste sea asumido para los fines pertinentes. La calidad en este sentido está fundamentada en diferentes aspectos que varían de acuerdo al contexto en el que se esté refiriendo. No es igual hablar de calidad de un producto, que de calidad de un servicio o que evaluar la calidad de un derecho como el de la educación. Por ello Bernal y otros (op. cit) exponen que la calidad se define teniendo en cuenta los fines propuestos y los alcances que se desean tener.

En el contexto educativo, de acuerdo con Nyathi, Kadhila y Aipanda, (2011, cp. Silva 2014):

la Calidad de la educación es un concepto multidimensional, multinivel y dinámico que se relaciona con las características contextuales del modelo educativo, la misión y los objetivos institucionales, así como con estándares específicos dentro de un programa, disciplina, institución o sistema (p. 11).

En los discursos de organismos internacionales, en particular el de la UNESCO (2005, 2008, 2013), se recalca que una educación de calidad debe posibilitar mucho más que la adquisición de competencias básicas, debe además ser inclusiva, efectiva y equitativa; en consecuencia, debe estar inserta en planes integrales amplios al servicio de la promoción socioeducativa de los grupos excluidos.

Ahora bien, al retomar los planteamientos de varios autores sobre la concepción de la calidad educativa, de acuerdo con Costa, (1996) señala que "no hay un consenso sobre la definición de calidad y menos aún sobre hasta qué punto y bajo qué modalidades es posible evaluarla". Así mismo Vidal (2007 cp. Orozco, Olaya y Villate 2009) señala que "la calidad plantea un campo de reflexión amplio y polémico, puesto que son polisémicos los significados que comporta y las tradiciones educativas que promueve" (p. 163)

De igual forma en los planteamientos de Martínez (2014, cp. Bernal y otros, 2015) el autor presenta la calidad educativa como una cuestión socialmente problematizada y sugiere que el Estado debería tomar posición frente a ellas para el diseño de sus políticas educativas. El autor expone desde un paradigma de la complejidad, que la calidad educativa requiere ser entendida de forma multidimensional y contextual. Así la calidad se presenta en un concepto más holístico donde se tienen en cuenta diferentes dimensiones y la diversidad de contextos para que esta sea más dinámica, abierta e incluyente.

Bernal y otros (2015) plantean que la calidad educativa en Iberoamérica, es medida a partir del impacto que han generado las políticas, sistemas, modelos, estándares, guías, procesos, pautas y otros aspectos relevantes, en los sistemas de gestión de la calidad y evaluación en las Instituciones Educativas.

Sin embargo, la tendencia en los últimos años de medición de la calidad educativa se realiza a partir de los resultados obtenidos por los estudiantes en pruebas externas e internas, haciendo de estos el único estándar de medida para las instituciones educativas con relación a los procesos académicos que se desarrollan dentro de las mismas y la incidencia de los actores involucrados.

Desde esta perspectiva, los indicadores y herramientas de medición de la calidad requieren ser más integrales para que no desconozcan las realidades contextuales, sociales y culturales de cada país.

De acuerdo con los planteamientos de Bernal y otros (op. cit) prescindir de los 
resultados de pruebas externas e internas como único indicador para medir la calidad es un tanto riesgoso, máxime en Colombia un país multicultural, con presencia de poblaciones vulnerables por la pobreza, el conflicto y el desplazamiento. De ahí que sea importante retomar otros indicadores que permitan evaluar la calidad de forma más integral y menos estandarizada.

De acuerdo con Martínez (2014), la calidad no se mide analizando hechos o respuestas aisladas sino todo un conjunto de iniciativas, acciones y propuestas en determinado contexto y momento histórico en la totalidad del sistema educativo. El planteamiento realizado por el autor pone de manifiesto una crítica congruente al sistema de evaluación de la calidad que se ha centrado solo en una parte del sistema. El concepto de calidad del autor, más incluyente, propone que la calidad se contemple de forma más holística, pudiendo reunir todos los actores y factores de un sistema.

Por su parte Carballo, Congosto y Fernández, (2013, cp. Bernal y otros, 2015) plantean que "una educación de calidad es aquella que pretende empoderar al estudiante, formar y motivar en él un pensamiento crítico y reflexivo con el uso creativo de técnicas pedagógicas que generen pertinencia y relevancia dentro del contexto educativo que vivencia". El planteamiento teórico de estos autores a nivel práctico pone de manifiesto un modelo educativo donde el estudiante participa activamente en la construcción de su conocimiento. Así, el estudiante es un sujeto crítico, que se cuestiona por las problemáticas de su entorno y a partir de ellas interpreta, propone y argumenta, construyendo conocimiento desde la realidad.

Este planteamiento es apoyado por Bernal y otros (2015) quienes conciben la educación de calidad a partir de los procesos que se vivencian dentro de las aulas escolares, el desarrollo de los planes y programas curriculares en un alto nivel, la formación integral que recibe, percibe y expresa el educando y el grado de competitividad alcanzado.

Esta nueva visión de la calidad que requiere trabajarse fuertemente, centra su mirada en el aula por ser el escenario donde se construyen los aprendizajes, se intercambian saberes y se forman los valores. Favorece no solo la formación integral, sino la diversidad y el respeto por la diferencia.

Una educación de calidad comprendida desde el aula, centra su mirada de forma más directa en lo que se vivencia en los procesos de enseñanza y su relación con los aprendizajes de los estudiantes. Visión que es apoyada por Aguerrondo (2014) cuando plantea que la educación es de calidad cuando la propuesta de enseñanza supone modelos de aprendizaje constructivo.

Así, cuando se habla de una educación de calidad es necesario centrar la mirada en lo que pasa en el aula con los procesos de enseñanza. Estudiar la enseñanza y la calidad de la misma, es necesario para determinar su incidencia en el aprendizaje de los estudiantes y de allí comprender diversas realidades que intervienen en el propósito de brin-dar una educación de la más alta calidad.

Para habar de calidad de la enseñanza es imprescindible comprender el concepto de enseñanza entendido desde diferentes autores.

De acuerdo con Guyot (2008, cp. Pogré 2012, p. 50), la enseñanza puede ser definida "como una práctica social compleja". Así, la enseñanza como práctica social tiene diferentes componentes que se adhieren al contexto, a los propósitos educativos, a los actores, entre otros y que por tanto no puede ser concebida de manera única, ni desarrollada de forma igual en los diferentes escenarios 
educativos.

Desde la perspectiva de Shulman (2011)

los fundamentos sobre la enseñanza se construyen en ideas que enfatizan en la comprensión y el razonamiento, la transformación y la reflexión. Cada uno de estos aspectos está estrechamente vinculado con el campo de la didáctica y propenden por mejorar la propuesta de la enseñanza como actividad y profesión.

Con relación a la didáctica, Aguerrondo (2014) señala que "la organización de la propuesta de enseñanza supone en primer lugar la intervención didáctica, es decir, lo que ocurre en el aula" (p. 10) La didáctica en relación con la enseñanza es uno de los espacios más críticos para el análisis de la calidad, porque allí se juega la transmisión y la generación del conocimiento.

Desde esta perspectiva, "la enseñanza ya no es todo gestión o acción docente, también es fundamental recuperar esa visión global de por qué, para qué, qué y cómo de la acción educadora" Murillo y Román (2011, p. 113). La enseñanza más allá del análisis de las actividades que desarrolla el docente e incluso de las didácticas como estrategias que facilitan el aprendizaje, es todo un "proceso" en el que es preciso regresar a la pedagogía como el conjunto de saberes que se ocupa de la educación como fenómeno típicamente social y específicamente humano.

Y más allá de la pedagogía se requiere enfatizar en la práctica pedagógica pues como diría Murillo y Román (2011) para valorar "el proceso de enseñanza" es necesario profundizar en lo que ocurre en el aula. Desde esta mirada, actualmente el mejoramiento de la educación en Colombia viene centrando la mirada en estudiar lo que sucede en el aula. Los procesos de enseñanza (métodos) y las prácticas de aula son el foco de las intervenciones para mejorar los resultados en el aprendizaje de los estudiantes.
Sobre los componentes de una buena enseñanza, según Shulman (2011) la enseñanza inicia con un acto de razón, continúa con un proceso de razonamiento, culmina con la acción de impartir, sonsacar, hacer participar, o seducir, y luego es objeto de mayores reflexiones hasta que el proceso puede reiniciársela (p. 17).

Ahora bien, hablar de calidad de la enseñanza implica según Litwin (1996), (cp. Granata 2000 p. 43), diferenciar entre el concepto de enseñanza exitosa y la buena enseñanza. La enseñanza exitosa remite a resultados acordes a los objetivos previstos, mientras que la "buena enseñanza" tiene un sentido moral que equivale a preguntar qué acciones docentes, basándose en principios morales, son capaces de provocar acciones de principio por parte de los alumnos.

La enseñanza de calidad retomando los aportes de Fenstermacher y Richardson (2005) es una enseñanza que a la vez reúne las características de la buena enseñanza y de la enseñanza exitosa. Es sensible a los estudiantes, porque se propone metas en relación con aspectos centrales del contenido disciplinar, y sensible a las estrategias que pone en práctica para lograrlo, pero que además consigue que los estudiantes tengan éxito en el aprendizaje.

En la actualidad otro concepto de enseñanza parece sobresalir y es la enseñanza efectiva. Pogré (2012, p. 26) plantea que "la investigación sobre enseñanza efectiva, en esencia, busca encontrar qué acciones del docente están asociadas al desempeño académico del estudiante". Los alcances de las investigaciones reflejan que la enseñanza efectiva se considera como aquella donde el docente implementa una serie de acciones y estas se ven reflejadas en los aprendizajes de los estudiantes.

Desde esta perspectiva la enseñanza requiere ser concebida desde un marco de 
referencia donde es importante que los docentes tengan bien cimentado su conocimiento disciplinar, el conocimiento didáctico de contenido y el pedagógico, desde esta integralidad las prácticas de aula se fortifican y se consolidan como referente para la promoción de un buen aprendizaje.

Al precisar sobre el papel relevante del docente en la enseñanza, Mejía, (2011) considera que la reconstrucción de la enseñanza de maestros y maestras, está en la transformación de la repetición y la memorización en prácticas diferentes que movilicen en los estudiantes las capacidades de observación, problematización y comprensión de contextos propios.

A nivel conceptual, la visión del autor sobre la enseñanza hace énfasis sobre la importancia de los métodos empleados por los docentes en la escuela para promover el aprendizaje de los estudiantes. La educación actual, requiere de prácticas de enseñanza cimentadas en métodos y técnicas por parte del maestro que promuevan el desarrollo de habilidades del pensamiento, donde el estudiante movilice mayores estructuras de metacognición.

En conclusión, cuando se habla de calidad de la enseñanza, uno de los principales protagonistas son los maestros, por lo cual será importante resaltar el significado que tiene la buena formación inicial y continua de los docentes y cual es en este sentido, la política de formación de maestros en Colombia.

En Colombia la enseñanza de la lectoescritura está fundamentada en una serie de lineamientos curriculares, estándares básicos de competencias y derechos básicos de aprendizaje que ilustran la forma sobre cómo desarrollar estos procesos en los estudiantes. También como parte de la política de gobierno a través del programa todos a aprender (PTA 2013 - 2018) en aras de favorecer la calidad de los procesos de enseñanza de la lectura y la escritura, se ha buscado cualificar la formación continua de los docentes desde el conocimiento disciplinar y el conocimiento didáctico de contenido en lectura y escritura.

En lenguaje, los vacíos más notables en formación de los docentes (tanto en lo urbano como en lo rural) están relacionados con elementos centrales de la didáctica en lectura y escritura, la génesis y adquisición del código escrito, así como las competencias que se requieren desarrollar en los procesos de comprensión y producción textual.

Desde el acompañamiento pedagógico del PTA a los docentes, estas dificultades son evidenciables tanto al momento de revisar las planeaciones de clases como al acompañar sus prácticas de aula. Asimismo, en las clases se evidencia las dificultades de los estudiantes para leer comprensivamente un texto $\mathrm{o}$ realizar una producción textual en diferentes asignaturas.

De igual forma, en diversos análisis realizados desde el PTA (2015 - 2017) a los resultados de los estudiantes en las pruebas saber, han dejado evidenciar como uno de los factores determinantes en el éxito de los estudiantes es la lectura comprensiva, esto ha llevado a concluir que la lectoescritura es un proceso básico para la calidad educativa, por cuanto es esencial para el desarrollo de los procesos del pensamiento que son fundamentales en el aprendizaje de cualquier otro contenido o asignatura.

En los últimos años, los sistemas educativos a nivel mundial, han fijado su interés en el desarrollo de políticas de calidad educativas centradas en el fortalecimiento de los procesos de enseñanza de la lectura y la escritura haciendo especial énfasis en los procesos de alfabetización. Sin embargo, la falta de unanimidad y de coherencia entre los gobiernos de diferentes países ha hecho que 
no se logren las metas esperadas. De ahí que la UNESCO (1993) planteara que el fortalecimiento de la lectura y la escritura deben ser parte de la política educativa a largo plazo más que de un ministro de turno.

En relación al tema de la habilidad de la lectura y la escritura para el año 1993, la UNESCO consideraba que había sido poco estudiado en América latina y el Caribe. Hoy en día se cuentan con más estudios donde se investiga a mayor profundidad la génesis de la lectura y la escritura a través de autoras como Teberosky y Ferreiro (1988), quienes vienen haciendo un gran aporte a los educadores de cómo enseñar estos procesos en la escuela.

Los trabajos de Ferreiro y Teberosky (1979), provenientes del campo de la psicología genética, demuestran que existe una psicogénesis del principio alfabético de escritura, independientemente de los métodos de enseñanza a los que el niño haya sido sometido. Los niños para aprender a leer y escribir, tal y como sucede con cualquier construcción cognitiva, pasan por diferentes niveles, formulando distintas hipótesis respecto a cómo se escribe, que van mejorando a medida que las ponen a prueba en distintos actos de escritura y se enfrentan a distintos conflictos cognitivos.

De acuerdo con Díaz (2000) las instituciones escolares han tenido como objetivo fundamental alfabetizar a los alumnos y este proceso comienza con la enseñanza de la lectura y la escritura en los primeros grados, donde se busca desarrollar las competencias básicas de la comunicación.

Desde esta perspectiva, en Colombia la lectura y la escritura en la escuela se ha limitado e instrumentalizado en prácticas de decodificación, prescindiendo de otros usos, como "procesos" que llevan a formas de construcción de sentidos, de interpretación y de la producción textual que se escapan a las formas escolares, al proyecto de sociedad y de individuo que se quiere inculcar (Sawaya, 2016).

Dentro del nuevo marco teórico de la enseñanza de la lectura y la escritura, la teoría de la lingüística los asume como proceso, esto implica que la lectoescritura es una actividad comunicativa continua del individuo en la sociedad y no simplemente un aprendizaje que se inicia en los primeros años (alfabetización), aunque es importante, abarca más que solo aprender a leer y escribir, sino que su objetivo es la adquisición de nuevos conocimientos, el desarrollo de sus saberes y su desempeño conforme se va encontrando con hechos de lectoescritura más complejos que requieran producir o interpretar textos orales o escritos que nunca había producido o interpretado antes.

Estas nuevas concepciones de la lectoescritura son sin duda un gran avance en las formas de pensar su enseñanza más allá de la simple decodificación (lectura) y de la transcripción (escritura). La lectoescritura desde esta perspectiva se concibe como el desarrollo de habilidades comunicativas donde se resalta la importancia que como habilidades sociales posibilitan la construcción de ciudadanía, democracia, valores, ejercer la participación, entre otros.

La lectura más allá de la tradición lingüística y de algunas teorías psicológicas, donde se considera el acto de "leer" como una decodificación del significado del texto, es considerada desde los lineamientos curriculares del MEN (1998) como:

Un proceso de interacción entre un sujeto portador de saberes culturales, intereses deseos, gustos, etcétera, y un texto como el soporte portador de un significado, de una perspectiva cultural, política, ideológica y estética particulares, y que postula un modelo de lector; elementos inscritos en un 
contexto: una situación de la comunicación en la que se juegan intereses, intencionalidades, el poder; en la que está presente la ideología y las valoraciones culturales de un grupo social determinado (p. 27).

Así, la lectoescritura según UNESCO (1993) requiere ser abordada desde su dimensión comunicativa y comprensiva. Este planteamiento, aunque del 1993, hoy cobra gran vigencia en el currículo del área de lenguaje, una vez que aún se privilegian los enfoques de enseñanza centrados en el estructuralismo: normativo/prescriptivo y se requiere una enseñanza centrada en el fortalecimiento de la significación donde "se haga evidente una serie de actuaciones o desempeños discursivos o comunicativos particulares asociados a las competencias lectoras y escritoras" (MEN, lineamientos curriculares, 1998, p. 28)

De acuerdo con los lineamientos curriculares (1998), anterior a su formulación, en Colombia los docentes del área de lenguaje concentraban su trabajo en la enseñanza de la lectoescritura en lo propiamente lingüístico, con una tendencia a lo normativo/prescriptivo: es decir, "enfatizando en la competencia lingüística, según los parámetros instruccionales de los libros de texto" (MEN, p. 56).

El enfoque normativo de la enseñanza de la lectoescritura se fundamentaba en el método deductivo y privilegiaba la memoria. En coherencia con lo anterior, de acuerdo con Jiménez y Guzmán, (2003 cp. Doria y Montes 2013) plantean "que la enseñanza debe organizarse siguiendo un orden preciso, determinado por consideraciones teóricas y prácticas que toman en cuenta las dificultades que plantea el aislar ciertos fonemas" (p. 27).

En la actualidad, los lineamientos curriculares disponen de un enfoque de enseñanza donde se espera que los docentes del área de lenguaje privilegien su estudio desde la significación y la enseñanza de la lectoescritura a partir de una perspectiva pragmática, en donde "se privilegie los modos de interactuar oralmente, de leer y de escribir de los estudiantes, fundamentos en la textolingüística" (MEN, 1998. p. 56) Así, en esta postura se concluye que se llega a las categorías gramaticales a través del análisis del uso.

En este nuevo enfoque, la lectoescritura es planteada como un proceso analítico, interactivo, constructivo y estratégico, que demanda según Sawaya, y Cuesta, (2016) "la construcción de un lector y escritor competente donde la escuela se transforme en un espacio de lectura y escritura haciendo hincapié sobre sus usos sociales" (p. 14).

Adicional a ello, la lectoescritura requiere dejar de ser vista solo como una tarea exclusiva del área de lenguaje, lo cual es corroborado por la UNESCO (1993) cuando plantea que la enseñanza de la lectoescritura está estrechamente vinculada con las demás asignaturas, donde los niños desde el principio interrogan los textos en su verdad de una manera crítica y creativa.

Ahora bien, al hacer una desagregación del binomio lectoescritura, para determinar las particularidades que encierra la enseñanza de la lectura y la escritura. De acuerdo con Josette (1993) aprender a leer, es desde el inicio, aprender a buscar el significado de un texto. Ya no es introducir letras o sílabas sin sentido para el lector.

Asimismo, sobre la enseñanza a partir del texto, retomando a Galaburri (2000), plantea que este permite preservar el sentido social de la lectura y la escritura, ya que los textos no son considerados como un material inerte que hay que imitar o decodificar, sino objetos de aprendizaje que cobran sentido y significado en los estudiantes, pues se lee y se escribe con la finalidad de comprender o ser 
comprendido por los otros.

Desde otro punto de vista, Lerner (2001) plantea que "la lectura aparece desgajada de los propósitos que le dan sentido en el uso social porque la construcción del sentido no es considerada como una condición necesaria para el aprendizaje". (p.120)

La postura de la autora, aunque refiere de más de 15 años atrás, confronta las realidades de la enseñanza de la lectura hoy en día donde la escuela sigue promoviendo prácticas de lectura anquilosadas en la sola decodificación y no logra despertar el interés ni la motivación de los estudiantes por leer y menos aún por asumir una postura crítica que permita a través de lo que lee construir subjetividades.

De acuerdo con Davidov y otros (1991) "la lectura no es un fin en sí, sino que debe iniciarse en el momento oportuno -que puede ser muy diferente de un niño a otro- en el proceso de reapropiación intelectual del mundo" (s/n). De ahí es importante considerar que para aprender a leer y a escribir más que señalar edades específicas, se requiere es aprovechar al máximo las motivaciones y necesidades de los niños y niñas por aprender hacerlo. De igual manera se espera que el docente pueda promover actividades espontaneas en el aula donde despierte y motive el interés del niño o la niña por el aprendizaje de la lectoescritura y no lo vea como una obligación.

Las principales problemáticas de hoy en Colombia en la enseñanza de la lectura, retomando a Lerner, (2001) podría deberse a que se enseña de una única forma a leer, por la ausencia de objetivos claros, a que se usa el mismo tipo de texto de lectura y se implementan las mismas estrategias.

Las concepciones y prácticas de enseñanza de la lectura de la actualidad poco difieren de las de hace 16 años donde se han privilegiado formas de leer rutinarias y poco motivantes para los estudiantes. Así mismo el uso del cuento es el único texto que ha estado presente en la escuela y se ha desperdiciado un sinnúmero de literatura que comprende el género narrativo y otros géneros discursivos.

Lerner (2001) señala que para que la lectura como objeto de enseñanza no se aparte demasiado de la práctica social que se quiere comunicar, es imprescindible "representar" o "re-presentar" en la escuela los diversos usos que ella tiene en la vida social. (p. 1)

De igual forma, esta posición es coherente con lo expuesto en los lineamientos curriculares en Colombia de 1998 donde el MEN, expone que:

Leer es un proceso de construcción de significados a partir de la interacción entre el texto, el contexto y el lector, en el cual el significado, a diferencia de lo que sostenía el modelo perceptivo motriz de la lectura, no está sólo en el texto, tampoco en el contexto ni en el lector, sino en la interacción de los tres factores, que son los que, juntos, determinan la comprensión (p. 47).

Con relación a los procesos de escritura de acuerdo con Hernández (2006) "la escritura puede considerarse como un proceso cognitivo complejo que consiste en traducir ideas, pensamientos y/o afectos en discurso escrito coherente con fines comunicativos determinados". En consecuencia, la escritura más allá de ser un proceso en el que se adquiere un código, tiene sentido en tanto se conciba su enseñanza en la escuela desde la significación, lo cual conlleva al desarrollo de diversas habilidades del pensamiento.

Por ello la enseñanza de la escritura retomando nuevamente a Hernández (op. cit). "requiere desarrollarse atendiendo a diferentes elementos: los tópico (¿qué escribir?), lo 
retórico (¿cómo escribirlo?) Y lo comunicativo-pragmático (¿para quién y para qué escribirlo?).

Desde esta perspectiva, la enseñanza de la escritura se enfoca en una escritura con sentido que tiene claro un propósito y una audiencia. Así la escritura permite al sujeto comprender el mundo $\mathrm{y}$ poder hacer representaciones del mismo.

De acuerdo con Brasil, (1997, cp. Sawaya 2016) la escritura debe ser un medio por el cual las personas logren una efectiva participación social. En este autor se amplía una de las funciones sociales que requiere promoverse a través de la enseñanza de la escritura, la cual debe promover la efectiva participación democrática y vincular al sujeto en la toma de decisiones. Desde esta percepción, la competencia escritora es concebida más desde el uso pragmático de la lengua que desde las estructuras sintácticas que solo dan forma al acto comunicativo.

En razón de lo pragmático, para Sawaya, (2016 p.10) "el aprendizaje de los usos sociales de la escritura se torna como una de las estrategias para la superación de la pobreza y del subdesarrollo", por ello la necesidad de proponer una enseñanza de la escritura que permita la expresión y comunicación de ideas y pensamientos que lleven a transformar las realidades y el mundo y no solo a transcribirlo y reproducirlo.

El punto de reflexión en este sentido, se centra en la necesidad de que en Colombia se transforme el enfoque de enseñanza actual de la escritura en la escuela, el cual tiene gran predominancia en la forma y no en el uso. Se asume así, un enfoque de escritura que trasciende del enfoque tradicional centrado en el manejo del código alfabético: fonema, sílaba y palabra, donde se ha puesto el énfasis en la relación sonido/grafía, por un enfoque en el que sobresale la significación, donde resulta necesario según el MEN "generar espacios de significación en los que la escritura cobre sentido social, y el problema de la fonetización y el acceso al código alfabético serán una necesidad que aparece de manera natural" (p. 30).

A propósito de ello, Jolibert, (1993) presenta una visión de la escritura planteando que el lenguaje escrito es constituido por textos y estos son ligados a situaciones reales de la vida, de uso contingente. Estos planteamientos son también apoyados por Van Dijk, (1995) gran expositor de la Lingüística textual como desarrollo objetivamente superador de la Gramática generativa, pero no necesariamente desvinculada de aquélla, ni mucho menos opuesta.

De ahí que sea necesario promover una enseñanza de la escritura centrada en la producción de textos, donde según Hernández (2006) se exija movilizar más habilidades del pensamiento y se parta de situaciones de contexto como un producto comunicativosocial. Esta concepción de escritura resalta la importancia de que la enseñanza de la escritura en la escuela permita ver la escritura como un acto de comunicación de ideas y de libre expresión.

Al respecto, Díaz Barriga y Hernández (2002) plantean que:

Es necesario enseñar en contexto los procesos escriturales y los géneros retóricos, sin omitir aspectos comunicativos y funcionales, en vez de enfocarse en los microprocesos (gramática, estructura, ortografía, morfosintaxis, puntuación) en los productos $\mathrm{y}$ en las evaluaciones superficiales (presentación, ortografía y gramática). (p. 89).

A nivel internacional, el planteamiento 
de estos autores tiene gran incidencia en las nuevas políticas de calidad para la enseñanza de los procesos de lectura y escritura, donde se viene privilegiando el uso de la lingüística textual con un enfoque semántico comunicativo por encima del estructuralismo que privilegia la gramática y la sintaxis de la lengua.

En Colombia, estas nuevas concepciones sobre los procesos de enseñanza de la lectura y la escritura, (por la experiencia obtenida como tutor en el área de lenguaje en varios Establecimientos Educativos de Antioquia en el PTA) son poco tenidas en cuenta en las instituciones puesto que de un lado en los planes de área de lenguaje muchos de estos fundamentos se desconocen y por el otro, los maestros desde las planeaciones de clase y las prácticas de aula se ha podido observar que la enseñanza de la lectura y la escritura siguen ancladas a los enfoques y métodos tradicionales. El estructuralismo y la gramática siguen siendo la meta principal de los docentes a la hora de direccionar sus prácticas en el aula.

Por ello, si se desea cualificar los procesos de enseñanza de la lectura y la escritura desde estos nuevos enfoques implica hacer seguimiento a la identificación de los factores que están incidiendo en la enseñanza de los procesos de lectura y escritura sea a nivel de formación docente, curricular, de planeación de clases, de materiales de enseñanza entre otros.

\section{CONCLUSIONES}

Es relevante que al hablar de mejorar la calidad de la enseñanza de la lectoescritura en Colombia, se profundice en las diversas concepciones que enmarcan las prácticas de enseñanza de la lectura y la escritura, que tienen como referente las últimas investigaciones y lineamientos curriculares realizados en este campo. Desde este propósito se podrá identificar no solo los elementos estructurantes de una buena enseñanza, sino también comprender las causas del distanciamiento que existe hoy entre la escuela, el maestro y las necesidades de los estudiantes en torno a la importancia de aprender a leer y a escribir.

El estudio de la calidad de la lectoescritura en Colombia ayudaría a tomar decisiones en el MEN sobre cómo fortalecer los procesos de formación situada de docentes en los aspectos relevantes (lineamientos, didácticas específicas, métodos de enseñanza, uso de materiales), que inciden en la calidad de este proceso. Además, ofrece la oportunidad de crear una política de formación docente en la educación inicial, que fortalezca aspectos desde el conocimiento disciplinar, pedagógico y didáctico de contenido en lectoescritura.

Construir un modelo teórico que explique los factores que inciden en la calidad de la enseñanza de la lectoescritura en la educación básica primaria, permitirá comprender la tarea y los retos que tienen los maestros encargados de la educación inicial en los niños y niñas para construir significativamente un proceso de aprendizaje óptimo de la lectura y el escritura.

La comprensión de las implicaciones que tiene la calidad en un buen proceso de enseñanza de la lectura y escritura posibilita que el maestro haga uso de mejores métodos y materiales educativos de enseñanza así como de la proyección de mejores ambientes de aprendizaje donde la lectoescritura parta de situaciones vivenciales y reales de comunicación.

\section{REFERENCIAS}

Aguerrondo, I. (2014). La calidad de la educación: Ejes para su definición y evaluación. Tomado de 
file://C:/Users/Ferney/Downloads/Lac alidaddelaeducacion.pdf

Bernal, S.D; Martínez, M.L; Parra, A.Y; Jiménez, J.L. (2015). Investigación Documental Sobre Calidad De La Educación En Instituciones Educativas Del Contexto Iberoamericano. En Revista EntramadosEducación y Sociedad, Año2, No. 2, Septiembre 2015 Pp. 107- 124

Costa, M. (1996). Aportes de las Ciencias del Lenguaje para la consideración de la calidad en Educación y su evaluación. Organización de Estados Iberoamericanos. Revista Iberoamericana de Educación

Davidov, V.V. y V.P. (1991) Perspectivas. Posiciones/controversias. Revista trimestral de educación

Díaz Barriga, F. y Hernández, G. (2002). Estrategias docentes para un aprendizaje significativo. Una interpretación constructivista. México DF: McGraw Hill

Doria, R., y Montes, A. (2013). Definición de un enfoque de enseñanza de la lectura y la escritura en el proceso de transición de la educación preescolar a la básica. Praxis, 9, 25 - 32

Fenstermacher, G.D. y Richardson, V. (2005). On making determinations of quality in teaching. Teachers College Record, 107(1), 186-213

Ferreiro, E., y Teberosky, A. (1979). Los sistemas de escritura en el desarrollo del niño, Méjico. Siglo veintiuno editores

Ferreiro, E., y Teberosky, A. (1982). Literacy before schooling. Heinemann Educational Books Inc., 70 Court St., Portsmouth, NH 03801

Granata M; Chada, M; Barale, C (2000). La enseñanza y la didáctica. Aproximaciones a la construcción de una nueva relación. Fundamentos en Humanidades, vol. I, núm. 1. Universidad Nacional de San Luis

Galaburri, M. (2000) (coord.) Proyecto de enseñanza para la formación de lectores y escritores. Historias de aula. Buenos Aires: Novedades Educativas

Guzmán, R. (2014). "Lectura Y Escritura. Cómo Se Enseña Y Se Aprende En El Aula." En: Colombia. Ed: Editorial
Universidad De La Sabana ISBN: 978-95812-0347-5

Hernández, G. (2006). Estudio sobre los modelos conceptuales y la autorregulación de la comprensión y la composición de textos: Un análisis de sus posibles relaciones. Tesis doctoral, Facultad de Psicología, UNAM, México

Hurtado, J. (2010) Metodología de la Investigación. Guía para la comprensión holística de la ciencia. Ediciones Quirón. Caracas, Venezuela

Instituto Colombiano para la Evaluación de la Educación (ICFES, 2016). Informe Pruebas SABER. Bogotá, Colombia

Johnson, D. W. y Johnson, R.T. (1999). Aprendizaje cooperativo y retroalimentación en instrucción de base tecnológica. En J.V. Dempsey y G.C. Ventas (Eds.), Instrucción interactiva y retroalimentación, pp. 133-157. Englewood Chiffs, NJ: Publicaciones de Educación Primaria

Jolibert, J. (1993). Recomendaciones para mejorar comprensión lectora y de Escritura de niños de áreas rurales y urbanas marginales. Proyecto principal de educación en América Latina y el Caribe. Lectoescritura factor clave de calidad de la educación. Boletín 32 Santiago de Chile

Lerner, D. (2001). Leer y escribir en la escuela: lo real, lo posible y lo necesario. México, Fondo de Cultura Económica. [Documento en línea]. Disponible en: www.cprtoledo.com/modules.php?op=m odload...file... [Consulta: 2009, diciembre 8]

Martínez F. (2014). Alternativas para evaluar la calidad social de la educación. Revista Iberoamericana de Educación (64/2). 114

Mejía M. (2011) Pensar la educación y la pedagogía en el siglo XXI. Universidad Pedagógica y Tecnológica de Colombia

Ministerio de Educación Nacional (MEN, 1998). Lineamientos curriculares en lengua castellana. Bogotá. Colombia. p. 28

MEN, (2013) Programa todos a aprender. Programa para la excelencia docente y 
académica"

Murillo, Román y Hernández (2011). Evaluación educativa para la justicia social. Revista Iberoamericana de evaluación educativa

Orozco, J. Olaya, A. y Villate, V. ¿Calidad de la educación o educación de calidad? Una preocupación más allá del mercado. Revista Iberoamericana de Educación, Núm. 51, septiembre-diciembre, 2009, pp. 161- 181 Organización de Estados Iberoamericanos para la Educación, la Ciencia y la Cultura España

Olivera y Orellana (2005) Escuchemos a Paulo Freire: una mirada a la educación popular. Revista electrónica diálogos educativos. Año 5, n 10, 2005 ISSN 07181310

Pogré, P. (2012). Formar docentes hoy, ¿qué deben comprender los futuros docentes? Perspectiva Educacional, Formación de Profesores, vol. 51, núm. 1, 2012, pp. 4556 Pontificia Universidad Católica de Valparaíso Viña del Mar, Chile

Sawaya, S y Cuesta, C. (2016). Las prácticas de lectura y escritura o el lugar del alumno como ciudadano. En Lectura y escritura como prácticas culturales. Universidad Nacional de La Plata. Editorial de la Universidad de La Plata 47 № 380 / La Plata B1900AJP / Buenos Aires, Argentina. 2016

Silva J, Bernal E, Hernández C (2014). Modelo de aseguramiento interno de la calidad para las instituciones de educación superior en el marco del mejoramiento continuo de la calidad de la educación superior en Colombia.

Shulman, L. (2011). Conocimiento y Enseñanza: Fundamentos de la Nueva Reforma. Vol. 9, Núm. 2

UNESCO, (1993). Proyecto principal de educación en América Latina y el Caribe. Lectoescritura factor clave de calidad de la educación. Boletín 32 Santiago de Chile UNESCO, (2011). La UNESCO y la Educación. "Toda persona tiene derecho a la educación"

Van Dijk, T. (1995). De la gramática del texto al análisis crítico del discurso. BELIAR (Boletín de Estudios Lingüísticos Argentinos), 2(6), 12-34 